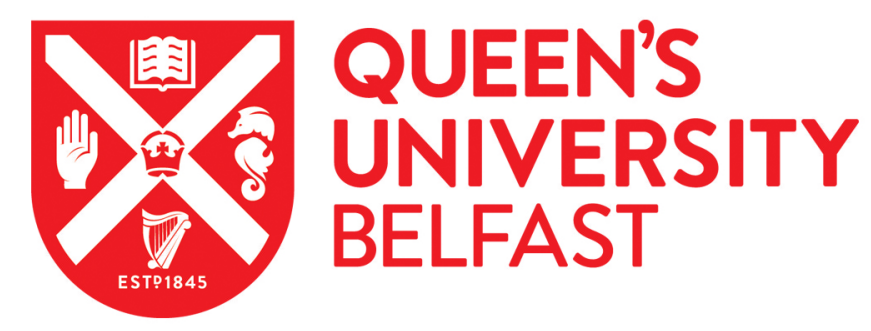

\title{
Circular array with displaced phase reference
}

Chepala, A., \& Fusco, V. (2019). Circular array with displaced phase reference. Microwave and Optical Technology Letters, 61(5), 1240-1243. https://doi.org/10.1002/mop.31740

\section{Published in:}

Microwave and Optical Technology Letters

\section{Document Version:}

Peer reviewed version

\section{Queen's University Belfast - Research Portal:}

Link to publication record in Queen's University Belfast Research Portal

\section{Publisher rights}

Copyright 2019 Wiley. This work is made available online in accordance with the publisher's policies. Please refer to any applicable terms of use of the publisher.

\section{General rights}

Copyright for the publications made accessible via the Queen's University Belfast Research Portal is retained by the author(s) and / or other copyright owners and it is a condition of accessing these publications that users recognise and abide by the legal requirements associated with these rights.

Take down policy

The Research Portal is Queen's institutional repository that provides access to Queen's research output. Every effort has been made to ensure that content in the Research Portal does not infringe any person's rights, or applicable UK laws. If you discover content in the Research Portal that you believe breaches copyright or violates any law, please contact openaccess@qub.ac.uk. 


\section{Title Page}

\section{Circular array with displaced phase reference.}

Running title: Chepala et al.

Authors: Anil Chepala and Vincent Fusco

Institutional Affiliation: Institute of Electronics, Communications and Information Technology (ECIT), Queen's University of Belfast, Belfast, BT3 9DT, UK; Corresponding author: achepala01@qub.ac.uk

Acknowledgments: This work was supported by a Queens's University of Belfast Studentship, and by EPSRC grants EP/N020391/1 and EP/P000673/1 


\title{
Main Text File
}

\begin{abstract}
We describe how the phase reference of a circular array can be re-located from its centre to an arbitrary radiating element located on the array while preserving the ability to project a collimated beam that can be steered into any azimuthal direction as with a conventional circular array. The ability to arbitrarily re-position the phase reference of the array makes this suitable for reducing Doppler effects when the array is in motion.
\end{abstract}

Key words: phase centre; circular array; Doppler

\section{INTRODUCTION}

A major advantage of the circular array is its ability to produce $360^{\circ}$ electronic beam scanning in azimuth without the variations in gain and far field radiation pattern shape when compared to a linear array [1]. This is achieved by setting a prescribed phase distribution according to [2]. This phase distribution is determined by selecting the geometric centre of the circular array as its phase centre. The purpose of this paper is to determine the possibility of maintaining $360^{\circ}$ circular array scanning functionality while simultaneously allowing the possibility of a controlled displacement of the array phase centre frame of reference. By shifting the effective radiation centre of the antenna in the opposite direction of displacement of the antenna whilst in motion, the technique helps compensate for the motion of the moving platform so that the antenna effectively appears stationary in space. This technique, could be for instance used to improve the performance of a moving-target-indicator radar. This approach results in narrowing of the spread of ground-clutter Doppler spectrum. Which in turn permits detection of low-velocity targets that would otherwise be clutter obscured [3].

\section{PHASE DISPLACED CIRCULAR ARRAY}

Consider, Fig. 1, which shows a circular array with element zero taken as the reference phase. In general, any element can be selected as the reference phase location.

A plane wave front $\mathrm{AB}$ arriving at element- 0 of, (in this example), a 16-element array, denoted CA-16, with uniform spacing at an angle $\theta$ relative to the reference axis. The phase shifts of the other elements are derived from the geometry to be in-phase along any selected angle $\theta$ with the signal received by element- 0 . If $\psi_{r}$ denotes the phase shift required by the $r^{\text {th }}$ element, then the equation set relating the required phase values for beam pointing along direction $\theta$ are given by equations (1)-(5).

$$
\begin{gathered}
\psi_{0}=0 \\
\psi_{1}=k l_{s} \sin (\alpha+\theta) \\
\psi_{2}=k l_{s} \sin (\alpha+\theta)+k l_{s} \sin (3 \alpha+\theta) \\
\psi_{3}=k l_{s} \sin (\alpha+\theta)+k l_{s} \sin (3 \alpha+\theta) \\
+k l_{s} \sin (5 \alpha+\theta) \\
\cdot \\
\cdot \\
\psi_{N-1}=k l_{s} \sin (\alpha+\theta)+k l_{s} \sin (3 \alpha+\theta)+ \\
k l_{s} \sin (5 \alpha+\theta)+ \\
\ldots \ldots \ldots .+k l_{s} \sin \{(2 N-3) \alpha+\theta\}
\end{gathered}
$$

Where $l_{s}$ is the length of the circle chord defining element-to-element separation. Examination of the series reveals that the phase shift for each element can be expressed as a phase shift of the previous element and an additional term. Incidentally, equations (1)-(5) form a major correction to those in [2]. The equations when further simplified 
can be expressed as shown in (6)-(10). This form shows that the shift in reference element is represented by the first term in (7)-(11), i.e. $k R \cos (\theta)$.

$$
\begin{gathered}
\psi_{0}=0 \\
\psi_{1}=k R \cos (\theta)-k R \cos (2 \alpha+\theta) \\
\psi_{2}=k R \cos (\theta)-k R \cos (4 \alpha+\theta) \\
\psi_{3}=k R \cos (\theta)-k R \cos (6 \alpha+\theta) \\
\dot{\psi_{N-1}}=k R \cos (\theta)-k R \cos \{2(N-1) \alpha+\theta\}
\end{gathered}
$$

The general equation for phase reference w.r.t element ' $p$ ' and the phases for the $r^{\text {th }}$ element can be represented as in equation (11). The term $k R \cos \left(\phi_{p}+\theta\right)$ represents the shifting of phase reference to arbitrary $p^{\text {th }}$ element and $k R \cos \left(\phi_{r}+\theta\right)$ represents the phase referred to array centre.

$$
\psi_{r}^{p}=k R \cos \left(\phi_{p}+\theta\right)-k R \cos \left(\phi_{r}+\theta\right)
$$

\section{SIMULATION RESULTS}

The circular array considered here is a 16-element array with element to element separation of one half wavelength, yielding an antenna to antenna pitch of $22.5^{\circ}$. The operating frequency is $900 \mathrm{MHz}$, each element is a balun-fed dipole antenna mounted horizontally one-quarter wavelength above a solid metal cylindrical ground plane. The diameter of the array is $85 \mathrm{~cm}$ and its depth is $50 \mathrm{~cm}$.

The phase distribution of each element is calculated from (11) for two different example cases with different angle of arrival ' $\theta$ '. Case-1 is with geometric centre as the phase reference and is plotted in Fig. 2a, Case-2 is with element -0 as the phase reference plotted is in Fig. $2 b$.These figures clearly show the effect of phase referencing on the phase distribution on the array.

Now let us consider two more cases:

Case-2a: Fixed reference element ' $p=4$ ' with different angle of arrivals ' $\theta$ '. The phase distribution for this case is obtained from equation (11) by fixing ' $p$ ' and varying ' $\theta$ '. This is plotted in Fig. 3a.

Case-2b: Fixed angle of arrival ' $\theta=0^{\circ}$ ' with different reference element ' $p$ '. The phase distribution for this case is obtained from equation (11) by fixing ' $\theta$ ' and varying ' $p$ '. This is plotted in Fig. $3 b$.

When the circular array elements are simulated with the phase distributions given in Fig. 2a, 2b and $3 \mathrm{a}, 3 \mathrm{~b}$ using the EM simulation software CST. The far-field amplitude pattern corresponding to distribution in Fig 2a is shown in Fig. 4. The far-field amplitude patterns corresponding to distribution in Fig $2 \mathrm{~b}$ is identical to that in Fig.4. Now when the array is excited with phase distribution given in case- 2a Fig.3a with element-4 as the phase reference for different angle of arrivals(AOA) $\theta$ of the plane wave, the far-field amplitude response is again virtually identical that in Fig.4, as are those for case-2b Fig.3b. In each case it is the actual phase distribution that is different.

Identical far field patterns in any direction $\theta$ can thus be obtained upon switching the reference element from one radiating element to any other element. This is done by phase referencing with respect to the element number under consideration i.e. by using the $p^{\text {th }}$ element as the reference element. Fig. 5 shows the far-field amplitude patterns for the array when element $0,4,8,12$ are alternatively used as the reference element while maintaining beam direction along $0^{\circ}$.

Therefore, by systematically changing the choice of reference radiating element, the phase reference of the array can be displaced. This when combined with appropriate beam selection updating can be used to make the array appear stationary and pointing towards a specified direction as it moves. The ability to displace the phase reference in any azimuthal direction while maintaining the far field pattern pointing into a fixed prescribed direction makes the approach we suggest in this paper suitable for applications where the antenna is mounted on a moving platform that can alter its track at any given time and where Doppler compensation, by virtue of array movement requires to be compensated. 


\section{CONCLUSION}

It has be shown that a circular array with selectable phase centre can be synthesised by relocating the phase reference of the array away from its geometrical centre to the location of an individual radiating element. Simultaneously it has been shown that it is possible to project a collimated far field pattern to any prescribed azimuthal direction. This means that the array when mounted on a moving platform can have its motion stabilised irrespective of its direction of its track in 2D. The resulting array could be used in radar systems for narrowing ground-clutter Doppler spectrum spread on vehicles such as drones that are likely to move along arbitrary tracks.

\section{REFERENCES}

1. B. Sheleg, "A matrix-fed circular array for continuous scanning," in Proceedings of the IEEE, vol. 56, no. 11, pp. 2016-2027, Nov. 1968

2. D. E. N. Davies and R. G. Fenby, "Series-fed circular array," in Electronics Letters, vol. 1, no. 9, pp. 264-265, November 1965

3. C. E. Muehe and M. Labitt, "Displaced-Phase Centre Antenna Technique," in Lincoln Laboratory Journal, Vol.12, No.2, pp 281-296, 2000

\section{Figures:}

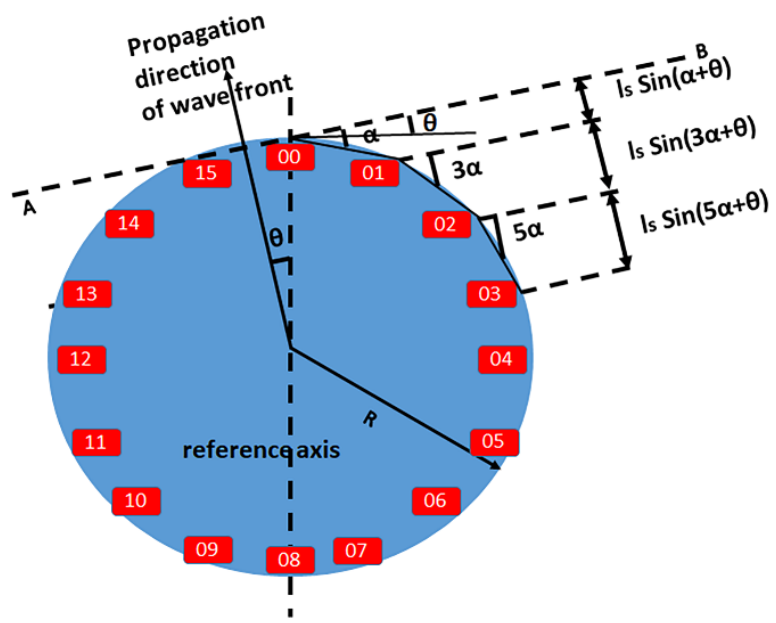

Figure 1 Circular array with phase reference to element centre. 


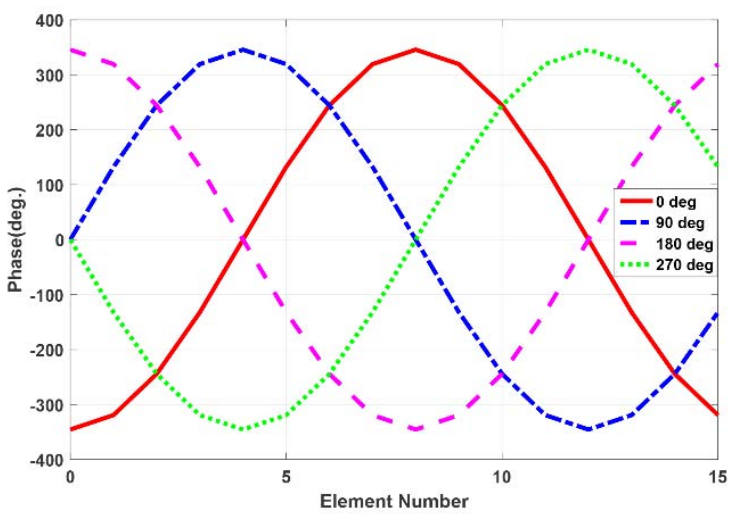

(a)

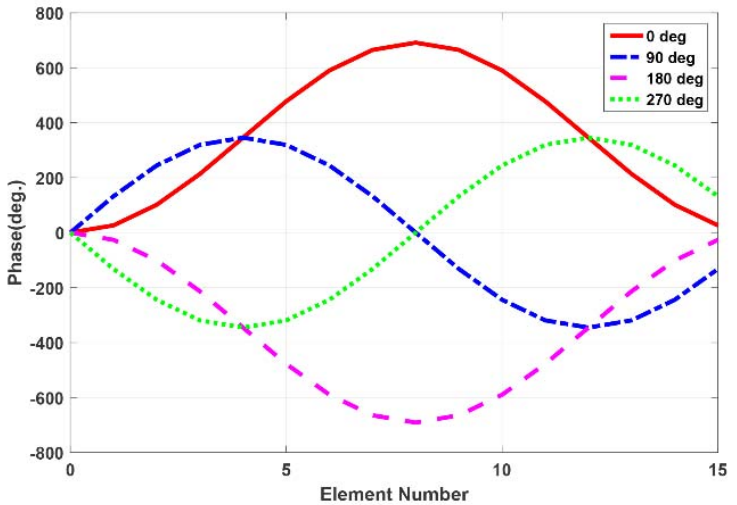

(b)

Figure 2 Simulated phase distribution on elements of the circular array.

(a) With its geometric centre as phase reference, (b) With element- 0 as phase reference. 


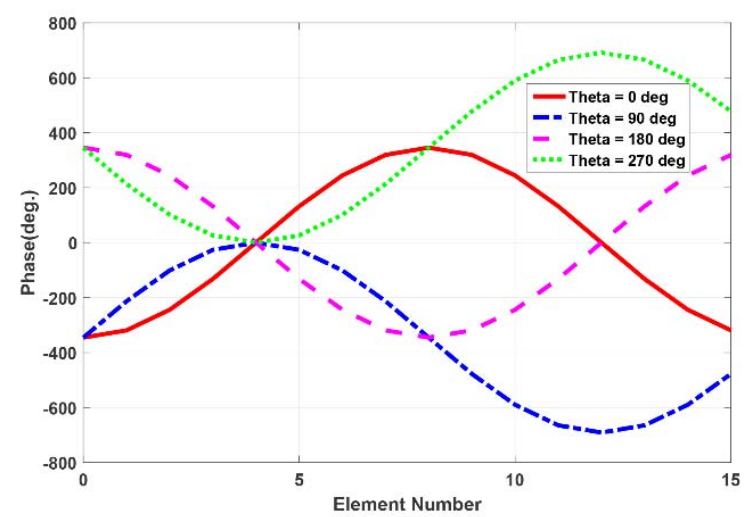

(a)

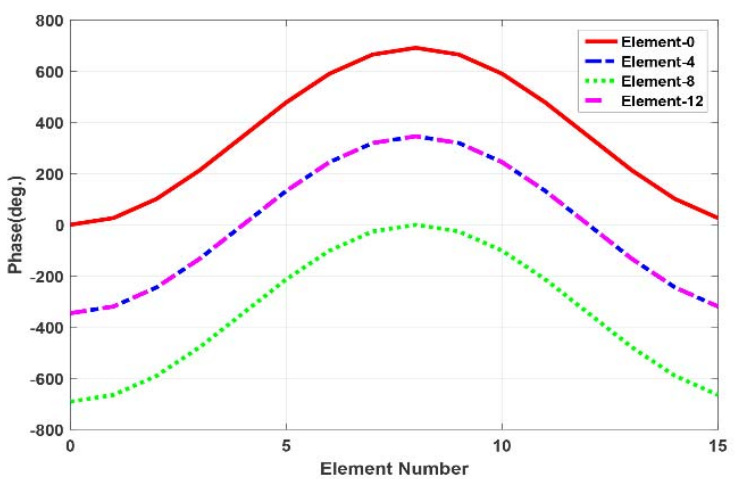

(b)

Figure. 3 Simulated phase distribution on elements of the circular array.

(a) Phase distribution for array with fixed reference element-4 for different angles of arrival, (b) Phase distribution for array with fixed angle of arrival $=0^{\circ}$ for different reference element.

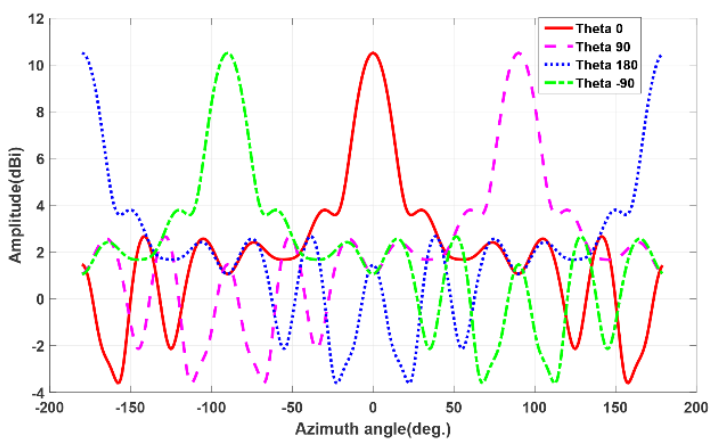

Figure. 4 Simulated farfield amplitude patterns for fixed reference element with changing AOA. 


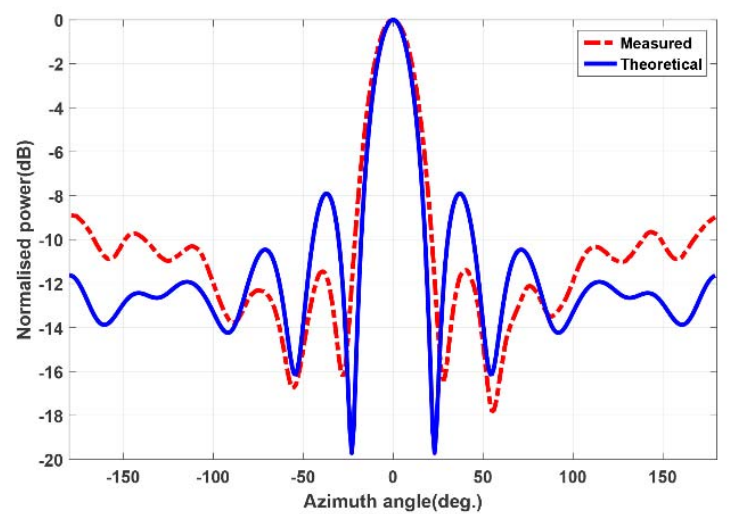

Figure. 5 Normalised farfield measured and simulated patterns for fixed AOA $0^{\circ}$ with changing reference elements. 